\title{
El control del tabaquismo y el movimiento de prevención
}

\author{
Joan R. Villalbí1,2, Rodrigo Córdoba ${ }^{1}$ \\ 1 Comité Nacional de Prevención del Tabaquismo \\ 2 Consell Asesor de Tabaquisme de Catalunya \\ Enviar correspondencia a:
}

Joan R Villalbí. Agència de Salut Pública de Barcelona. PI Lesseps 1. 08023 Barcelona. jrvillal@aspb.es

\section{RESUMEN}

Este editorial revisa los antecedentes de la legislación de prevención del tabaquismo que está en curso en España a finales de 2005: para vencer las resistencias de la industria tabaquera se precisa de un esfuerzo organizado. Se repasa la historia del movimiento de prevención en España, su organización a través del Comité Nacional de Prevención del Tabaquismo (CNPT), su estrategia, y el trabajo que ha permitido alcanzar esta situación en nuestro país, que puede representar un punto de inflexión. Los esfuerzos del CNPT se configuraron en tres ejes: dar una voz más fuerte y coherente al movimiento de prevención ante los medios de comunicación, definir las políticas apropiadas en diversos campos importantes para la prevención, y ejercer una interacción constante con los poderes públicos, especialmente el Ministerio de Sanidad y Consumo y los miembros españoles del Parlamento Europeo. Los progresos se fundamentan en la mejora de la coordinación del movimiento (ampliando sus miembros y estableciendo canales de comunicación), y en la relación con el movimiento europeo de prevención. La experiencia del movimiento de prevención del tabaquismo permite extraer lecciones para otros problemas de salud relacionados con sustancias adictivas en los que las políticas públicas son importantes.

Palabras clave: tabaquismo; prevención; políticas; organización.

$\mathbf{E}$ stos últimos meses, se han sentado las bases para el desarrollo en España de políticas de prevención del tabaquismo efectivas, culminando los esfuerzos que durante años han desarrollado muchos profesionales. La ley de medidas sanitarias frente al tabaquismo, los cambios fiscales orientados a vincular el impuesto sobre el tabaco con los presupuestos sanitarios, y el flujo de recursos hacia las comunidades autónomas para actuar en este campo plantean probablemente un punto de inflexión en nuestro país. Para que esto sea una realidad, ha hecho falta un largo camino, y especialmente el desarrollo de un esfuerzo deliberado por influir en las políticas

\section{ABSTRACT}

This editorial reviews the antecedents to the legislation on preventing smoking which is in place in Spain at the end of 2005. An organised effort is necessary in order to overcome the resistance of the tobacco industry. It also reviews the history of the prevention movement in Spain, its organisation through the National Committee for the Prevention of Smoking (NCPS), its strategy and the work which led to the current situation in this country which could represent an inflexion point. The work by the Committee was configured around three axles; to give a stronger and more coherent voice to the anti-smoking movement with regard to the media, to define the appropriate policies in diverse fields of importance to prevention, and to exercise a constant interaction with public powers, in particular with the Ministry of Health and Consumer Affairs and the Spanish Members of the European Parliament. Progress was based on an improvement in the coordination of the movement (increasing its membership and establishing communication channels) and in relation to the European prevention movement. The experience of the smoking prevention movement allows certain lessons to be learned for dealing with other health problems relating to addictive substances where public policies are important.

Key words: smoking; prevention; policies; organisation. públicas con el que el movimiento de prevención del tabaquismo ha alcanzado su mayoría de edad.

La ciencia demostró de forma incontrovertible el daño que produce el tabaco en la salud hace ya unos 50 años. Más recientemente se ha demostrado el daño que produce el humo ambiental de tabaco (HAT). Este conocimiento se ha trasladado a la sociedad, de modo que en los países desarrollados se ha reducido mucho el consumo de tabaco, y se han adoptado regulaciones que favorecen esta reducción, mediante políticas como las fiscales, o el control de la publicidad $^{1}$. La industria tabaquera se ha resistido a este 
proceso, y están bien documentados sus esfuerzos por influir en los políticos, a veces a los científicos, y sobre todo manipular a los medios de comunicación de modo que no se vieran afectados sus intereses -también en España². Mientras que algunos países han conseguido incorporar políticas de prevención a la acción del gobierno, acelerando así el proceso de cambio de la epidemia tabáquica, otros han tenido menos éxito en este campo, y progresan más lentamente. Hasta hace poco, ésta era la situación española. La emergencia de un movimiento organizado ha contribuido a cambiar esta situación.

\section{POLÍTICAS DE PREVENCIÓNY SOCIEDAD}

En efecto, en las sociedades desarrolladas, la sociedad civil juega un papel fundamental en el proceso para avanzar en el control del tabaquismo ${ }^{3}$. El esfuerzo por traducir los conocimientos sobre el tabaco y el daño que causa en políticas de prevención choca con obstáculos importantes. Para vencer estas resistencias se precisa una presión constante, imposible sin un cierto grado de organización ${ }^{4}$. En una sociedad democrática, el apoyo social permite superar inercias y resistencias a la implantación de políticas públicas. Sin él, las presiones de los grupos de interés (como la industria tabaquera) sobre el gobierno frenan el progreso: presiones que se ejercen directamente o a través de los medios de comunicación, en nuestro caso presentando el control del tabaquismo como algo social y políticamente controvertido, y que tendría impactos negativos sobre el empleo o la recaudación fiscal.

En nuestro país, es visible la creciente implicación de la sociedad civil en la demanda de políticas de control del tabaquismo. Su principal componente son diversas organizaciones relacionadas con profesionales de la sanidad -que son los primeros actores implicados en todos los países- pero también hay otros agentes ${ }^{5}$. Éstos son las organizaciones de víctimas del tabaco o afectados por su uso (como los laringectomizados), las organizaciones no gubernamentales de tipo altruista que no son primariamente de profesionales de la salud (como las asociaciones y ligas contra el cáncer), las organizaciones como nofumadores.org formadas por ciudadanos no vinculados a las profesiones sanitarias, y otras con fines diversos pero que por su naturaleza o principios pueden mantener posiciones favorables a la prevención, como las de consumidores.

\section{EL MOVIMIENTO}

No existe en España una tradición de movimiento por la temperancia, contrario al consumo de tabaco y alcohol. El que hubo históricamente, vinculado al naturismo y a los ideales anarquistas, fue destruido tras la guerra de 1936-39. La difusión pública del descubrimiento científico del daño que causaba el tabaco a la salud no desencadenó en España respuestas organizadas pro prevención en los años sesenta por el contexto político y social de la dictadura y por la propiedad estatal de la empresa monopolística de tabaco en aquel momento (Tabacalera) ${ }^{6}$. Con timidez se difunde en los ambientes médicos, en los que el consumo de tabaco es por entonces muy elevado. Tras el cambio democrático, desde mediados de los años ochenta van emergiendo personalidades vinculadas a las profesiones sanitarias y a las administraciones públicas o a la red asistencial de financiación pública. No puede hablarse propiamente aún de un movimiento de prevención, y la mayoría de las personalidades que expresan de forma visible posiciones respecto al tabaco son expertas en un tema u otro, pero sin una visión global. La conciencia de la necesidad de coordinación independiente de la administración se va consolidando a principios de los años noventa, a medida que se amplían las iniciativas de prevención, creando las condiciones para la articulación de una organización global, que será el Comité Nacional para la Prevención del Tabaquismo (CNPT) 7 .

\section{LA ORGANIZACIÓN}

Las bases para la creación del CNPT se sientan en una reunión informal de los participantes españoles en la $9^{\text {a }}$ Conferencia Internacional sobre el tabaco y la salud de París en octubre de 1994. En su curso, se constata la débil presencia española y la frustración por los escasos progresos conseguidos desde la anterior Conferencia de 1992 en Buenos Aires, proponiendo crear una plataforma de coordinación. En una reunión en Toledo se concretan los principios sobre los que se fundará, y en 1996 se constituye formalmente y se registra al amparo de la ley de asociaciones. El CNPT se constituye básicamente como una alianza o coalición de organizaciones ya existentes, que mantienen su actividad de prevención del tabaquismo, y que inicialmente se circunscribe a sociedades científicas o de profesionales sanitarios (entre ellas Socidrogalcohol, tan relacionada con esta revista). Aunque acepta socios individuales, la toma de decisiones gravita sobre los representantes de las asociaciones miembros, lo que se refleja en sus estatutos, su organización y su financiación, así como en el ámbito en que prioriza sus actividades. Tras unos inicios dubitativos con cierta dispersión, el CNPT inicia un proceso a largo plazo orientado influir en las políticas públicas relacionadas con el tabaco. Para ello, se concentra en 
introducir el tabaco en la agenda política y definir de forma integral las acciones de prevención prioritarias en España. Este esfuerzo se concreta inicialmente en la elaboración del Libro Blanco sobre el tabaquismo en España ${ }^{8}$. Este documento, aún muy consultado hoy en la web del CNPT, compila toda la información dispersa existente hasta entonces, incluyendo los primeros documentos de síntesis en español sobre cuestiones como el tabaquismo pasivo. Además, formula una batería de propuestas para la prevención. El Libro Blanco marca un hito: moviliza los esfuerzos de muchas personas clave, sintetiza la información, define prioridades, circula ampliamente, llega a la prensa...

Posteriormente el CNPT contribuye a generar documentos de estrategia sobre otros aspectos clave como la publicidad o la fiscalidad, a difundir la necesidad de influir en las políticas públicas entre el movimiento de prevención, y mediante seminarios y expertos desarrolla documentos de posición sobre políticas clave: espacios sin humo, publicidad, fiscalidad, tratamiento y las presuntas campañas para jóvenes 'de prevención' generadas por la propia industria tabaquera. Para ello se esfuerza en reforzar la organización del movimiento de prevención, consolidando su secretaría, ampliando el número de miembros, estableciendo relaciones estables con otros actores, construyendo una web y desarrollando una infraestructura de comunicación electrónica mediante listas de correos (de agentes clave, periodistas interesados, políticos...) además de la que propone la UICC mediante Globalink, que en este período incrementó notablemente el número de miembros residentes en España: una docena en 1996, más de un centenar en 2005.

En definitiva, los esfuerzos del CNPT se configuran en tres ejes: dar una voz más fuerte y más coherente al movimiento de prevención ante los medios de comunicación, definir las políticas apropiadas en diversos campos importantes para la prevención pero alejados de la medicina, y ejercer un esfuerzo de interacción constante con los poderes públicos, especialmente el Ministerio de Sanidad y Consumo y los eurodiputados españoles en el Parlamento Europeo. Para progresar en estos ejes, se basa en dos instrumentos: mejorar la coordinación del movimiento en España (ampliando sus miembros y estableciendo canales de comunicación), y engarzar con el movimiento europeo a través de su participación en la Red Europea para la Prevención del Tabaquismo (REPT, en sus siglas en inglés ENSP).

\section{RESULTADOS}

Los cambios favorables en el control del tabaquismo registrados en los últimos años no pueden atribuirse únicamente a la acción del CNPT, pero parece indudable que éste ha jugado un cierto papel en conseguirlos. Quizás lo más destacable es el cambio en el clima de los medios de comunicación respecto al tabaco, que han modificado su perspectiva, al difundirse cada vez más las políticas efectivas para su control, así como la comprensión de las tácticas de la industria tabaquera ${ }^{9}$. En buena parte, esto es atribuible al hecho de que se ha estabilizado un abanico estable de personas cualificadas como portavoces del movimiento de prevención con criterio sobre todas las políticas relevantes. Esto se ha constatado en estudios de los medios de comunicación ${ }^{10}$.

EI CNPT ha influido en la adopción de normativas en España, contribuyendo a hacerlas más preventivas en su interacción con el Ministerio, tanto para mejorar la normativa sobre el tabaco en los medios de transporte en 1999 como la trasposición de la Directiva de Productos en 2002. Asimismo, el CNPT o sus miembros han sido consultados por diversas Comunidades Autónomas para la construcción de planes autonómicos sobre el tabaco. Y especialmente desde el CNPT se han producido acciones de lobbying para el control del tabaquismo ante los miembros españoles del Parlamento Europeo para las Directivas de Publicidad en 1998 y 2002, y las Directivas de Productos y de Fiscalidad discutidas en 2001-02. También se hicieron acciones ante los miembros del Consejo Interterritorial de Salud con vistas a la aprobación en enero del 2003 del Plan Nacional de Prevención y Control del Tabaquismo propuesto por la ministra Ana Pastor. Y desde finales de 2004 se ha desarrollado una actuación febril tendente a concretar las acciones reguladoras de dicho Plan en una ley que permitiera un salto cualitativo, aprovechando la trasposición legal de la directiva europea de publicidad. Esto se ha conseguido con la ley presentada por la ministra Elena Salgado que está finalizando actualmente su trámite parlamentario en un clima de amplio consenso político y mediático. Esta ley comporta una regulación rigurosa de la publicidad y la implantación de la prohibición general de fumar en los lugares de trabajo, con algunas excepciones en el sector de hostelería, restauración y ocio, y comporta un gran salto adelante. El trabajo del CNPT organizando al movimiento de prevención y articulando su presencia ante los medios de comunicación y el Ministerio, así como su labor de contacto directo con los diversos grupos políticos parlamentarios y con muchos de los miembros de la Comisión de Sanidad del Congreso que interviene decisivamente en estos trámites ha sido crucial. Si inicialmente la propuesta fue acogida con cierta hostilidad por la oposición y con controversias por algunos aliados parlamentarios del gobierno, el producto final ha sido su aprobación por unanimidad en la Comisión, con algunas mejoras. 


\section{UNA PERSPECTIVA DE FUTURO}

Valoramos que durante estos años el CNPT se ha legitimado y reforzado como referente para los medios de comunicación y como interlocutor exigente, leal y realista de las administraciones públicas para la prevención del tabaquismo. Ha mantenido su independencia ante gobiernos de diverso signo, preservando lo esencial de su misión: avanzar en la prevención de los estragos que causa el tabaco. La aprobación de la ley Salgado marca probablemente un hito irreversible y el fin de una fase. El movimiento de prevención se ha institucionalizado más en España (el CNPT es interlocutor habitual del Ministerio) y también ha logrado cierta consolidación organizativa (con un Congreso anual, una revista, canales de comunicación electrónicos...). Los retos de futuro para el CNPT son mantener su independencia para poder ejercer su papel estimulando la adopción de políticas de prevención. El CNPT debe mantener relaciones de colaboración con la industria farmacéutica, que colabora habitualmente con las sociedades médicas y promueve el tratamiento farmacológico de los fumadores. Pero debe preservar también su independencia, para poder impulsar otras políticas de prevención que se han mostrado importantes para el control del tabaquismo en la experiencia internacional (impuestos sobre el tabaco, regulación de la publicidad, espacios sin humo...). Por otra parte, el CNPT debe ampliar la alianza en pro de la salud con nuevos actores. Para ello debe seguir incorporando las organizaciones vinculadas a la salud y las profesiones sanitarias, pero también otros agentes ajenos al campo sanitario pero con los que comparte valores y principios. Entre ellos están las organizaciones de pacientes y víctimas del tabaco, el movimiento de consumidores que ha tenido un papel creciente en este campo, el movimiento sindical, el movimiento por la defensa del medio ambiente y el de protección de la infancia. Y el nuevo contexto planteará la necesidad de crear plataformas comparables al CNPT con un campo de acción autonómico, escenario crucial en el futuro para las políticas de prevención.

\section{REFERENCIAS}

1 Villalbí JR, López V. La prevención del tabaquismo como problema político. Gac Sanit 2001; 15: 265-72.

2 Granero L, Villalbí JR, Gallego R. ¿Quién se opone a la prevención? Un mapa de los actores pro-tabaco en España. Gac Sanit 2004; 18: 374-9.

3 Joosens L. Estrategias de presión para el avance de políticas de prevención del tabaquismo. En: Villalbí JR, Ariza C (Coords). El tabaquismo en España: situación actual y perspectivas para el movimiento de prevención. Barcelona: Comité Nacional de Prevención del Tabaquismo y Sociedad Española de Salud Pública y Administración Sanitaria, 2000; 23-28.

4 Villalbí JR, López V. La prevención del tabaquismo como problema político. Gac Sanit 2001; 15: 265-72.

5 Granero L. Las políticas de control y prevención del tabaquismo en España y los actores a favor y en contra del tabaco. Tesina del programa de Maestría en Gestión Pública. Barcelona: Institut d’Educació Contínua de la Universitat Pompeu Fabra, 2002.

6 Villalbí JR. El tabaco como problema de salud pública. En: Cabasés JM, Villalbí JR, Aibar C. Invertir para la salud: prioridades en salud pública. Informe SESPAS 2002. Valencia: Generalitat Valenciana y Sociedad Española de Salud Pública y Administración Sanitaria, 2002; 113129

7 Villalbí JR. Trabajando para el control del tabaco desde el Comité Nacional de Prevención del Tabaquismo. Prev Tab 2001; 3(Supl 1): S16-17.

8 Becoña E (Coord). Libro blanco sobre el tabaquismo en España. Barcelona: Glosa, 1999.

9 Escolà C. Philip Morris creó un lobby en España. El País 1360, Domingo 23 de enero del 2000; págs 30-31.

10 Observatorio de la Comunicación Científica y Médica de la Universitat Pompeu Fabra. Medicina, comunicación y sociedad. Informe Quiral 1999. Barcelona: Rubes Editorial, 2000; 121. 